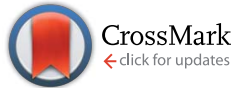

Cite this: RSC Adv., 2016, 6, 58566
Received 1st May 2016 Accepted 11th June 2016

DOI: 10.1039/c6ra11265

www.rsc.org/advances

\section{Lighting up the gold nanoparticles quenched fluorescence by silver nanoparticles: a separation distance study $\dagger$}

\author{
Shuang Li, ${ }^{\text {ac }}$ Taishi Zhang, ${ }^{\text {ab }}$ Zhijun Zhu, ${ }^{a}$ Nengyue Gao ${ }^{a}$ and Qing-Hua Xu*ab
}

Contrast ratios of "turn-on" fluorescence probes determine the detection sensitivity of fluorescence-based assays and detections. Conventional fluorescence turn-on methods are generally based on recovery of quenched fluorescence to its initial intensity. Here we designed a nano-platform in which the fluorescence intensity of a pre-quenched fluorophore was enhanced up to the level significantly higher than the intensity of the unquenched fluorophores by utilization of plasmon coupling enhanced fluorescence. In this approach, the fluorescence of rhodamine B isothiocyanate (RiTC), which was prequenched by gold nanoparticles (Au NPs), was subsequently enhanced by silver nanoparticles (Ag NPs) upon formation of $\mathrm{AgCSiO}_{2}-\mathrm{Au}-\mathrm{RiTC}$ nanostructures. The thickness of $\mathrm{SiO}_{2}$ spacer shell can be adjusted to tune the gap size between Au NPs and Ag NPs to control the plasmon coupling strength to achieve the optimum fluorescence enhancement. Among the series of $\mathrm{AgCSiO}_{2}-\mathrm{Au}-\mathrm{RiTC}$ NPs with $\mathrm{SiO}_{2}$ shell thickness of $4,10,13,20,25,36,43$ and $50 \mathrm{~nm}$, the optimum florescence of the coupled nanostructure was found to occur at silica shell thickness of $13 \mathrm{~nm}$ with its fluorescence intensity 101 times that of the Au-RiTC NPs, 4.4 times that of free RiTC. In addition to plasmon enhanced excitation efficiency and quantum yields due to metal-chromophore interactions, huge local electric field amplification the gap region induced by plasmon coupling between Au NP and Ag NPs contributes additionally to further enhance the excitation efficiency to achieve the observed fluorescence enhancement. This plasmon coupling enhanced fluorescence concept should offer a new design strategy to give rise to a "turn-on" fluorescence responses with improved signal-to-background ratios.

\section{Introduction}

Fluorescence-based assays and detections are important research tools for various applications such as chemical and biological sensing, ${ }^{1}$ live-cell imaging, ${ }^{2}$ rapid diagnosis, ${ }^{3}$ flow cytometry, ${ }^{4}$ and DNA sequencing, ${ }^{5}$ owing to its high sensitivity, simplicity, and low cost. ${ }^{6-8}$ The detection sensitivity of fluorescence based methods can be improved by increasing the contrast ratio by either increasing detectable fluorescence

${ }^{a}$ Department of Chemistry, National University of Singapore, 3 Science Drive 3, Republic of Singapore 117543. E-mail: chmxqh@nus.edu.sg

${ }^{b}$ NUS Graduate School for Integrative Sciences and Engineering, National University of Singapore, Republic of Singapore 117456

'State Key Laboratory of Luminescent Materials and Devices, South China University of Technology, Guangzhou 510640, P. R. China

$\dagger$ Electronic supplementary information (ESI) available: Two-photon photo-luminescence and extinction spectra of Au NPs and Au-RiTC NPs; optimization of molar ratio of Au NP to RiTC; TEM images and normalized extinction spectra of $\mathrm{Ag} @ \mathrm{SiO}_{2}$ NPs with various silica shell thicknesses; FTIR spectra of $\mathrm{Ag@SiO} \mathrm{O}_{2}$ before and after MPTMS condensation; emission spectra and two-photon luminescence of coupled nanostructures; DTT treatment of $\mathrm{Ag} @ \mathrm{SiO}_{2}-\mathrm{Au}-\mathrm{RiTC} \mathrm{NPs}$ dispersion; fluorescence lifetime measurement; simulation results; fluorescence enhancement in $\mathrm{Ag} @ \mathrm{SiO}_{2}-\mathrm{RiTC}$ NPs. See DOI: 10.1039/c6ra11265j signal strength or reducing the non-specific background level using fluorescence quenchers. Gold nanoparticles (Au NPs) have been known as efficient fluorescence quenchers, 9-10 orders of magnitude more efficient than typical small molecule dye-quencher pairs. ${ }^{9}$ Lots of activatable probes have been designed to detect the analytes by using dye-Au NP pairs..$^{10-12}$ However, the contrast ratios of most of dye-Au NP based assays rely on the difference between reduced background fluorescence quenched by Au NPs and recovered fluorescence to its initial intensity by forcing Au NP and dye molecules apart. Further improved signal-to-background ratio could be achieved if the reduced fluorescence could be enhanced to the level higher than that of initial fluorescence intensity, which could be realized by metal-enhanced fluorescence.

Noble metal NPs, such as Au and Ag NPs, exhibit unique optical properties known as localized surface plasmon resonance (SPR). ${ }^{13-16}$ Interactions between metal NPs and fluorophores in close proximity could either quench ${ }^{17,18}$ or enhance $^{19}$ the fluorescence of chromophores through the modification of its excitation efficiency and fluorescence quantum yield. ${ }^{20}$ This phenomenon has attracted intense attention in both fundamental studies and practical applications. ${ }^{21}$ Most studies on metal enhanced fluorescence are based 
on silver island films or silver nanoparticle overlayers on the glass substrates. ${ }^{22-25}$ Fluorescence enhancement of tens of folds was obtained in these studies. ${ }^{24,25}$ However, it is very difficult to achieve fluorescence enhancement by using metal NPs in the colloid solution. ${ }^{19,26,27}$ Plasmon coupling interactions between closely spaced metal NPs have been known to result in a significantly enhanced local electric field within the gap region, ${ }^{28,29}$ which is responsible for giant enhancement in surface enhanced Raman scattering (SERS). ${ }^{30}$ Plasmon coupling between adjacent metal NPs could also be utilized to increase the excitation efficiency and radiative decay rates, thereby enhancing the overall fluorescence of nearby chromophores. ${ }^{13,31}$ Smart design on coupled nanostructures is of great interest to achieve large fluorescence enhancement. Fluorescence enhancement in the coupled nanostructures has been investigated on substrate, ${ }^{32}$ ensemble solutions, ${ }^{33,34}$ and on single molecule level $\mathrm{l}^{\mathbf{2 0 , 3 5 , 3 6}}$ based on various assembly methods such as electrostatic interactions, molecular interactions, bond formation and lithography. Previous reports demonstrated 1340-fold enhancement in emission intensity of TPQDI in a bowtie nanoantenna structure, ${ }^{20} 170$-fold of Atto-665 in aggregated Ag NPs, ${ }^{37} 470$-fold of Atto-665 in a tip-to-tip Au nanorod dimer, ${ }^{38}$ 2530 -fold of an IR dye-labelled streptavidin in aggregated $\mathrm{Ag}$ NPs, ${ }^{39}$ and 7400 -fold of IgG in plasmonic nanodot arrays. ${ }^{40}$

In this work, we designed a nano-platform (Scheme 1) in which the fluorescence intensity of a pre-quenched fluorophore was enhanced to the level of a few times higher than the original intensity of the unquenched fluorophores by utilization of plasmon coupling enhanced fluorescence. The fluorescence of a fluorophore rhodamine B isothiocyanate (RiTC) was prequenched by $\mathrm{Au}$ NPs upon formation of Au-RiTC NPs, which

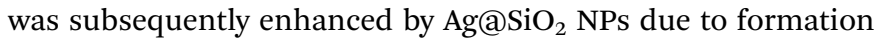
of $\mathrm{Ag} @ \mathrm{SiO}_{2}-\mathrm{Au}-\mathrm{RiTC}$ composite nanostructures. The coupled nanostructures were prepared by conjugation of Au NPs and $\mathrm{SiO}_{2}$ coated Ag NPs via gold-sulfur interaction. The thickness of $\mathrm{SiO}_{2}$ spacer shell can be adjusted to tune the gap size between $\mathrm{Au}$ NPs and Ag NPs to investigate the effects of plasmon coupling strength on the fluorescence enhancement. The optimum florescence of the coupled nanostructure was found to occur at silica shell thickness of $13 \mathrm{~nm}$ with its fluorescence intensity 101 times that of dyes quenched by Au NPs, 4.4 times that of free dyes. This plasmon coupling enhanced fluorescence concept should offer a new design strategy to give rise to a "turnon" fluorescence response with improved signal-to-background ratios.

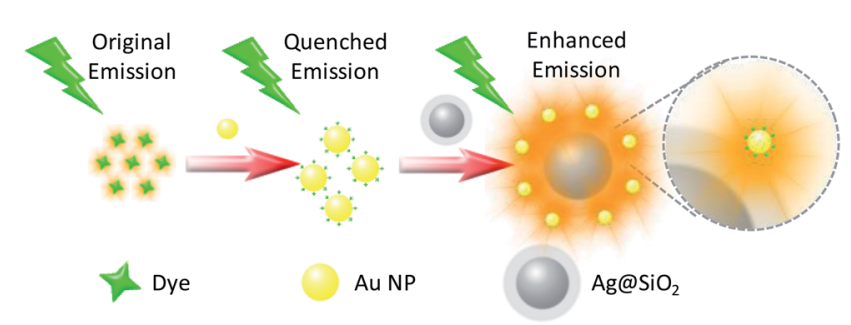

Scheme 1 Schematic illustration of plasmon coupling induced fluorescence enhancement of the chromophore that is pre-quenched by Au NPs.

\section{Experimental}

\section{Materials and instrumentation}

Silver nitrate $\left(\mathrm{AgNO}_{3}\right)$, hydrogen tetrachloroaurate(III) trihydrate $\left(\mathrm{HAuCl}_{4} \cdot 3 \mathrm{H}_{2} \mathrm{O}\right)$, tri-sodium citrate dehydrate, tetraethoxysilane (TEOS), sodium dodecyl sulfate (SDS), DL-dithiothreitol (DTT), sodium hydrate $(\mathrm{NaOH})$, rhodamine B isothiocyanate (RiTC), polyvinylpyrrolidone (PVP, MW 40 000), (3-mercaptopropyl)trimethoxysilane (MPTMS), (3-amino-propyl)trimethoxysilane (APTMS) were purchased from Sigma Aldrich. All solvents are analytical grade and used as received without further purification. All aqueous solutions are prepared in de-ionized water.

Absorption and fluorescence spectra were measured on a Hitachi UH5300 UV-vis spectrophotometer and a Horiba Jobin-Yvon FluoroMax-4 Spectrophotometer, respectively. Transmission electron microscopic (TEM) images were taken on a JEOL 2010 microscope operating at $200 \mathrm{kV}$. Fourier transform infrared (FTIR) measurements were performed by using an Excalibur 3100 FT-IR spectrometer (Varian, USA) in the transmission mode at room temperature.

\section{Preparation of Au NPs and Au-RiTC NPs}

$13 \mathrm{~nm}$ Au NPs were prepared by using a modified Turkevich method. ${ }^{41}$ Briefly, an aqueous solution of $\mathrm{HAuCl}_{4}(50 \mathrm{~mL}, 1.0$ $\mathrm{mM}$ ) was first heated to boiling under stirring before addition of tri-sodium citrate $(5.0 \mathrm{~mL}, 38.8 \mathrm{mM})$ in water. The mixture solution was further boiled for $15 \mathrm{~min}$. The solution was slowly cooled to room temperature after the color changed from pale yellow to deep red, indicating formation of Au NPs. The obtained Au NPs had an average diameter of $(13.0 \pm 1.1) \mathrm{nm}$ based on the TEM images. The concentration of as-prepared Au NPs colloid solution was determined by measuring their extinction at $520 \mathrm{~nm}\left(\varepsilon=2.7 \times 10^{8} \mathrm{~L} \mathrm{~mol}^{-1} \mathrm{~cm}^{-1}\right) .{ }^{42}$

Citrate stabilized Au NPs are extremely prone to aggregation due to weak interactions between citrate ions and Au NPs. SDS was therefore utilized to stabilize Au NPs. SDS solution $(100 \mu \mathrm{L}$, $1 \mathrm{wt} \%$ ) was added into $1.0 \mathrm{~mL}$ as-prepared $\mathrm{Au}$ NPs before centrifugation (13 $200 \mathrm{rpm}, 15 \mathrm{~min}$ ) to remove extra SDS and citrate. Different amounts of freshly prepared RiTC solution $\left(100 \mu \mathrm{M}, \mathrm{H}_{2} \mathrm{O}\right)$ were added into the above dispersion and stirred at room temperature overnight. The Au-RiTC NPs dispersions were subsequently diluted to a total volume of $1.0 \mathrm{~mL}$ and kept in fridge at $4{ }^{\circ} \mathrm{C}$ for further use.

\section{Preparation of Ag NPs and $\mathrm{Ag} @ \mathrm{SiO}_{2}$ NPs}

$\mathrm{Ag}$ NPs were prepared by using the polyol process developed by Xia and co-workers. ${ }^{43}$ Briefly, ethylene glycol $(5.0 \mathrm{~mL})$ was heated in an oil bath to $160{ }^{\circ} \mathrm{C}$ before addition of $\mathrm{AgNO}_{3}$ solution $(0.12 \mathrm{M}, 3.0 \mathrm{~mL})$ and PVP in ethylene glycol solution (0.18 $\mathrm{M}, 3.0 \mathrm{~mL}$ ) simultaneously under vigorous stirring. The mixture was kept at $160{ }^{\circ} \mathrm{C}$ for $1 \mathrm{~h}$ under continuous stirring before cooling down to the room temperature. The obtained Ag NPs were purified by centrifugation (30 $\mathrm{min}, 9000 \mathrm{rpm})$ and then redispersed into ethanol. The Ag NPs were further purified by $3000 \mathrm{rpm}$ centrifugation for $30 \mathrm{~min}$ to remove Ag NPs with smaller sizes and $20 \mathrm{~min}$ at $500 \mathrm{rpm}$ to remove those with larger 
sizes. The mean size of the obtained Ag NPs is $(81.3 \pm 16.9) \mathrm{nm}$ in diameter.

$\mathrm{Ag} @ \mathrm{SiO}_{2} \mathrm{NPs}$ were prepared by following a modified Stöber method. ${ }^{44} \mathrm{Ag}$ NPs solution (1.0 mL, $0.5 \mathrm{nM}$ in ethanol) was injected into the mixture of ethanol $(19.0 \mathrm{~mL}), \mathrm{H}_{2} \mathrm{O}(5.0 \mathrm{~mL})$ and $\mathrm{NaOH}(250 \mu \mathrm{L}, 0.1 \mathrm{M})$ under stirring. Different amounts of TEOS ( $1 \%$ in ethanol) were subsequently injected three times at a 30 min interval under continuous gentle stirring to initiate the formation of $\mathrm{SiO}_{2}$. The reactions proceeded at room temperature for $24 \mathrm{~h}$. A silica layer formed on the surface of Ag NPs through hydrolysis and condensation of silane. The thickness of $\mathrm{SiO}_{2}$ layer was controlled by the amount of TEOS. The obtained $\mathrm{Ag} @ \mathrm{SiO}_{2} \mathrm{NPs}$ were isolated by centrifugation at $8000 \mathrm{rpm}$ for 3 min, washed with ethanol several times, and then re-dispersed in ethanol for further use.

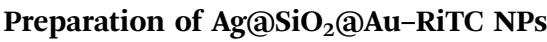

$\mathrm{Ag} @ \mathrm{SiO}_{2}$ NPs were first functionalized with sulfhydryl (-SH) groups using MPTMS following a previous report. ${ }^{45}$ Briefly, 30

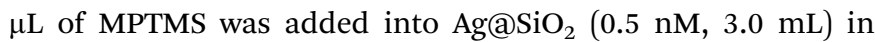
ethanol solution and kept for $45 \mathrm{~min}$ under stirring. The mixture solution was heated to $80{ }^{\circ} \mathrm{C}$ and then kept for $2 \mathrm{~h}$ under a nitrogen atmosphere. The $\mathrm{Ag} @ \mathrm{SiO}_{2} \mathrm{NPs}$ were then washed three times with absolute ethanol and kept in fridge for further use. The functionalization with $-\mathrm{SH}$ was confirmed by FTIR.

To couple Au-RiTC NPs and Ag@SiO ${ }_{2}-\mathrm{SH}$ NPs, mixtures of aliquots of Au-RiTC NPs (10 nM in term of Au NPs) in $\mathrm{H}_{2} \mathrm{O}$ with different amounts of $\mathrm{Ag} @ \mathrm{SiO}_{2}-\mathrm{SH} \mathrm{NPs}\left(0.4 \mathrm{nM}, \mathrm{H}_{2} \mathrm{O}\right)$ were rotated $(300 \mathrm{rpm})$ in the dark at room temperature overnight and diluted to a concentration of $0.5 \mathrm{nM}$ (in terms of Au NPs) prior to use.

\section{Preparation of $\mathbf{A g @ S i O}{ }_{2}-\mathrm{RiTC}$ NPs}

$\mathrm{Ag} @ \mathrm{SiO}_{2} \mathrm{NPs}$ were first modified with $-\mathrm{NH}_{2}$ groups through hydrolysis and condensation of APTMS. Briefly, a solution of APTMS (100 $\mu \mathrm{L}, 25 \% \mathrm{v} / \mathrm{v}$, chloroform) was added into $\mathrm{Ag@SiO}$ NPs $(10 \mathrm{~mL}, 0.8 \mathrm{nM})$ in ethanol. The mixture reacted overnight under stirring at room temperature. The as-prepared $\mathrm{Ag} @ \mathrm{SiO}_{2}-$ $\mathrm{NH}_{2}$ NPs were isolated by centrifugation, washed with ethanol several times, and then dispersed in ethanol prior to use. The successful surface functionalization can be verified by the change of zeta-potential of silica surface from negative to positive. Ag@SiO 2 -RiTC NPs were subsequently prepared by mixing RiTC solution $\left(0.3 \mu \mathrm{M}, \mathrm{H}_{2} \mathrm{O}\right)$ with different amounts of $\mathrm{Ag} @ \mathrm{SiO}_{2}-\mathrm{NH}_{2} \mathrm{NPs}$ in $\mathrm{H}_{2} \mathrm{O}$. The mixture was kept rotating overnight before the measurements.

\section{Results and discussion}

The strategy for plasmon coupling enhanced fluorescence of a pre-quenched fluorophore is shown in Scheme 1. Rhodamine B isothiocyanate (RiTC) was chosen as the model fluorophore because the absorption and fluorescence spectra of RiTC have reasonable overlap with the extinction spectrum of metal NPs (Fig. 2a) to ensure resonance interactions. RiTC is a water- soluble chromophore with strong affinity to $\mathrm{Au} .^{46}$ The fluorescence quantum yield of RiTC in methanol is $\sim 0.7 .{ }^{47}$ Plasmon enhanced fluorescence of rhodamine molecules have been previously reported in the silver nanoparticles overlayers. ${ }^{\mathbf{2 4 , 2 5}} \mathrm{It}$ is generally difficult to achieve plasmon enhanced fluorescence in the colloid solution. Here we utilize plasmon coupling induced additional excitation enhancement to achieve fluorescence enhancement by using coupled $\mathrm{Ag@SiO}{ }_{2} \mathrm{NPs}$ and $\mathrm{Au}-$ RiTC NPs. The extinction spectrum of Au-RiTC NPs is approximately a superposition of absorption spectrum of RiTC and extinction spectrum of Au NPs. The relative molar ratio between $\mathrm{Au}$ NPs and RiTC molecules has been optimized to ensure efficient quenching of the RiTC fluorescence (Fig. S1 and S2 $\dagger$ ). $\mathrm{Au}-\mathrm{RiTC}$ NP with approximately $\sim 600$ RiTC molecules in each nanoparticle was used in the subsequent studies, in which the fluorescence of Au-RITC NPs is about 23 times quenched compared to that of the same amount of free RiTC molecules in solution. The significantly quenched fluorescence of RiTC in $\mathrm{Au}$-RiTC NPs is due to energy transfer from RiTC to the Au NP owing to good spectral overlap between absorption spectrum of Au NPs and emission spectrum of RiTC (Fig. 1). ${ }^{11,48}$

$\mathrm{SiO}_{2}$ coated Ag NPs (Ag@SiO ${ }_{2} \mathrm{NPs}$ ) was utilized to light up the fluorescence of Au-RiTC NPs. Ag NPs generally display larger fluorescence enhancement effects than Au NPs. ${ }^{31}$ Larger sized metal NPs generally have higher scattering-to-absorption ratios, which are beneficial for metal enhanced fluorescence. ${ }^{49}$ $\mathrm{Ag}$ NPs with a diameter of $81 \pm 17 \mathrm{~nm}$ are therefore prepared to act as the metal core. Thickness of $\mathrm{SiO}_{2}$ shell plays a critical role in the lighting-up effect. On the one hand, $\mathrm{SiO}_{2}$ shell will be utilized to optimize the separation distance between RiTC molecules and Ag NPs since metal enhanced fluorescence is strongly dependent on the metal-chromophore separation distance. On the other hand, plasmon coupling interactions between $\mathrm{Au}$ NP and Ag NP also strongly depend on their separation distance. The overall fluorescence intensity of the coupled nanostructures can be optimized by adjusting the thickness of $\mathrm{SiO}_{2}$ shell. Upon coating with $\mathrm{SiO}_{2}$ shell, the SPR band of $\mathrm{Ag}$ NPs in $\mathrm{H}_{2} \mathrm{O}$ displayed a gradual redshift owing to higher refractive index of silica than that of aqueous media. ${ }^{50}$ The redshift increased to $c a .20 \mathrm{~nm}$ when the silica shell thickness increased to $50 \mathrm{~nm}$ (Fig. S3†). TEM images (Fig. 2 \& $\mathrm{S} 4 \dagger$ ) confirm the formation of $\mathrm{Ag} @ \mathrm{SiO}_{2}$ NPs with uniform $\mathrm{SiO}_{2}$ shells. Ag@SiO $\mathrm{NPs}_{2}$ was further surface-modified with thiol
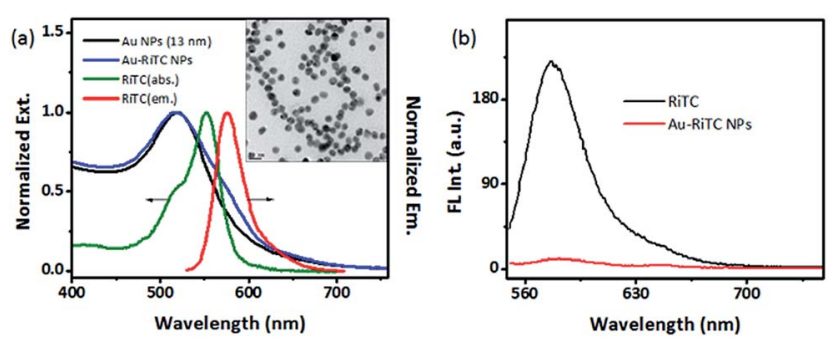

Fig. 1 (a) Normalized extinction spectra of Au NPs (13 nm), RiTC, and $\mathrm{Au}-\mathrm{RiTC} N P s$ in $\mathrm{H}_{2} \mathrm{O}$; (b) fluorescence of RiTC $(0.3 \mu \mathrm{M})$ with and without Au NPs $(0.5 \mathrm{nM})$. The inset in (a) is TEM images of Au NPs (13 nm). 


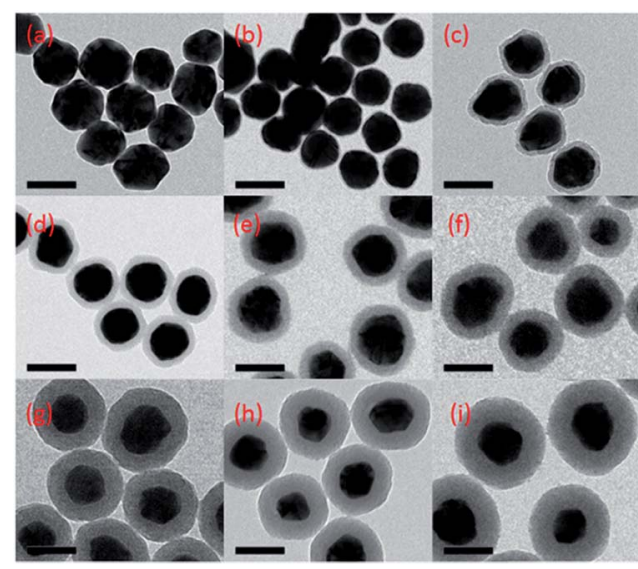

Fig. 2 TEM images of Ag NPs (a) and $\mathrm{Ag} \mathrm{SSiO}_{2} \mathrm{NPs}$ with silica shell thickness of $4 \mathrm{~nm}$ (b), $10 \mathrm{~nm}$ (c), $13 \mathrm{~nm}$ (d), $20 \mathrm{~nm}$ (e), $25 \mathrm{~nm}$ (f), $36 \mathrm{~nm}$ (g), $43 \mathrm{~nm}$ (h) and $50 \mathrm{~nm}$ (i). Scale bars in all images are $100 \mathrm{~nm}$.

groups by condensation of siloxane with MPTMS. Successful attachment of thiol groups onto the $\mathrm{Ag} @ \mathrm{SiO}_{2} \mathrm{NPs}$ were verified by the FTIR analysis (see Fig. S5†).

$\mathrm{Ag} @ \mathrm{SiO}_{2}-\mathrm{Au}-\mathrm{RiTC}$ NPs were formed by attaching $\mathrm{Ag} @ \mathrm{SiO}_{2}$ NPs and Au-RiTC NPs via the gold-sulfur interactions. Comparison of the extinction spectrum of $\mathrm{Ag} @ \mathrm{SiO}_{2}-\mathrm{Au}-\mathrm{RiTC}$ NPs (blue lines) and the superposition of the extinction spectra of its individual components (Ag@SiO ${ }_{2}$ NPs + Au-RiTC NPs) showed decreased extinction in the short wavelength region and simultaneously increased extinction at the longer wavelength region (Fig. 3), which is a signature of plasmon coupling between Au-RiTC NPs and $\mathrm{Ag} @ \mathrm{SiO}_{2}$ NPs. Formation of $\mathrm{Ag} @ \mathrm{SiO}_{2}-\mathrm{Au}-\mathrm{RiTC}$ NPs can be directly visualized by their TEM images (Fig. 3 inset). As plasmon coupled noble metal nanoparticles generally give strong two-photon photoluminescence, ${ }^{\mathbf{4 8 , 5 1 , 5 2}}$ plasmon coupling interactions between the $\mathrm{Ag}$ NP core and surrounding Au NPs within each $\mathrm{Ag} @ \mathrm{SiO}_{2}-\mathrm{Au}-$ RiTC NPs were further confirmed by significantly increased twophoton photoluminescence of $\mathrm{Ag} @ \mathrm{SiO}_{2}-\mathrm{Au}$-RiTC NPs upon the attaching Au-RiTC NPs to the surface of $\mathrm{Ag@SiO}{ }_{2}$ NPs (Fig. S6†).

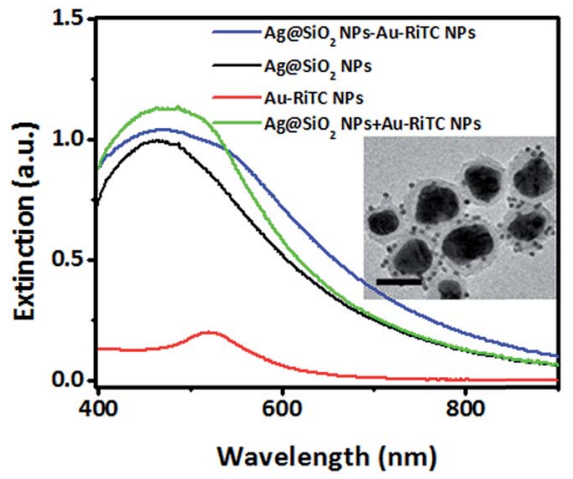

Fig. 3 Extinction spectra of $\mathrm{Ag} \mathrm{CSiO}_{2} \mathrm{NPs}, \mathrm{Au}-\mathrm{RiTC} \mathrm{NPs}, \mathrm{Ag} \mathrm{aSiO}{ }_{2}-$ $\mathrm{Au}-\mathrm{RiTC}$ NPs (coupled), AgaSiO $2 \mathrm{NPs}+\mathrm{Au}-\mathrm{RiTC}$ NPs (non-coupled). The $\mathrm{SiO}_{2}$ thickness is $13 \mathrm{~nm}$. Inset is the TEM image of $\mathrm{Ag}\left(\mathrm{aSiO}_{2}-\mathrm{Au}-\right.$ RiTC NPs.
The results of lighting up of the quenched fluorescence of Au-RiTC NPs by Ag@SiO ${ }_{2}$ NPs are shown in Fig. 4. Fig. 4a shows the change in fluorescence spectra of Au-RiTC NPs upon gradual addition of $\mathrm{Ag} @ \mathrm{SiO}_{2} \mathrm{NPs}$ with $\mathrm{SiO}_{2}$ shell thickness of 13 $\mathrm{nm}$. Upon gradual addition of $\mathrm{Ag} @ \mathrm{SiO}_{2} \mathrm{NPs}$, the fluorescence intensity of Au-RiTC NPs under excitation at $530 \mathrm{~nm}$ steadily increased until reaching a plateau (Fig. 4a inset). Upon formation of $\mathrm{Ag} @ \mathrm{SiO}_{2}-\mathrm{Au}-\mathrm{RiTC} \mathrm{NPs}$, the fluorescence of Au-RiTC NPs was found to be enhanced by up to 101-fold, which is 4.4 times enhanced compared to that of the same amount of free RiTC molecules. The possible contribution of the observed fluorescence recovery/enhancement due to release of RiTC molecule into the solution during the experiments can be excluded by various control experiments (Fig. S7 $\dagger$ ). The fact that fluorescence intensity of $\mathrm{Ag} @ \mathrm{SiO}_{2}-\mathrm{Au}-\mathrm{RiTC}$ NPs is a few times larger than that of free RiTC molecules confirms the vital role of plasmon coupling in the observed fluorescence enhancement, since fluorescence intensity increase due to release of RiTC cannot go beyond that of free RiTC molecules. Fluorescence lifetime measurements (Fig. S8 $\dagger$ ) have been performed to understand the origin of the observed fluorescence enhancement. The fluorescence lifetime of free RiTC molecules in $\mathrm{H}_{2} \mathrm{O}$ was measured to be $\sim 1.5$ ns. Upon binding to Au NPs, the fluorescence lifetime of Au-RiTC NPs was shorter than the instrument response function of $\sim 100$ ps and cannot be accurately determined. After attaching Au-RiTC NPs to the surface of $\mathrm{Ag} @ \mathrm{SiO}_{2} \mathrm{NPs}$, the fluorescence lifetime of $\mathrm{Ag} @ \mathrm{SiO}_{2}-\mathrm{Au}-\mathrm{RiTC}$ NPs with thickness of $13 \mathrm{~nm}$ remained shorter than the instrument response function $(\sim 100 \mathrm{ps})$ instead of increasing back to $1.5 \mathrm{~ns}$. The observation of significantly enhanced fluorescence intensity and short fluorescence lifetime at the same time further confirm that the observed fluorescence increase is due to the plasmonic interactions between Au-RiTC NPs and $\mathrm{Ag} @ \mathrm{SiO}_{2}$ NPs rather than release of RiTC molecules.

$\mathrm{Ag} @ \mathrm{SiO}_{2}-\mathrm{Au}-\mathrm{RiTC} \mathrm{NPs}$ with a series of shell thicknesses (4, $10,13,20,25,36,43$ and $50 \mathrm{~nm}$ ) have been prepared to optimize the fluorescence enhancement and investigate the underlying enhancement mechanisms. The extent of optimum fluorescence enhancement was found to strongly depend on the thickness of $\mathrm{SiO}_{2}$ shell (Fig. $4 \mathrm{~b}$ and $\mathrm{S} 9 \dagger$ ), which controls the separation distance between Au-RiTC NPs and Ag NP core.
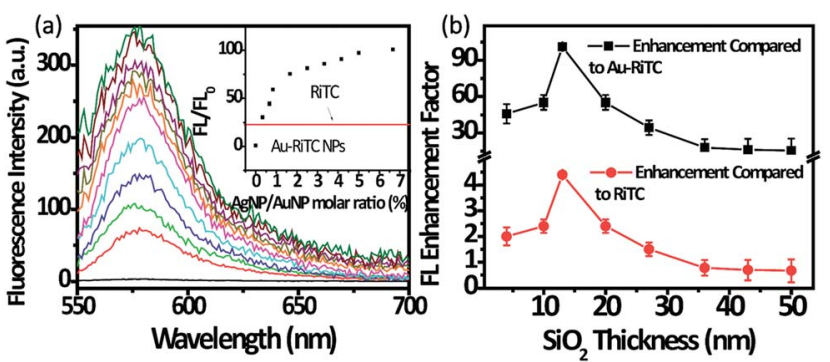

Fig. 4 (a) Fluorescence spectra and intensities of Au-RiTC NPs upon gradual addition of $\mathrm{AgOSiO}$, with $\mathrm{SiO}_{2}$ thickness of $13 \mathrm{~nm}$. The red line in the inset is the intensity level of free RiTC; (b) fluorescence enhancement factors of $\mathrm{Ag}\left(\mathrm{CSiO}_{2}-\mathrm{Au}-\mathrm{RiTC}\right.$ NPs with different $\mathrm{SiO}_{2}$ shell thicknesses compared to Au-RiTC NPs and free RiTC molecules. 
Among a few shell thickness studied in our work, the optimum fluorescence enhancement for the $\mathrm{Ag} @ \mathrm{SiO}_{2}-\mathrm{Au}-\mathrm{RiTC}$ NPs was observed at the $\mathrm{SiO}_{2}$ shell thickness of $13 \mathrm{~nm}$. Less fluorescence enhancement was found for shell thickness either less or larger than $13 \mathrm{~nm}$.

Fluorescence intensity of chromophores is proportional to the product of excitation efficiency and fluorescence quantum yield, both of which will be modulated by metal-chromophore interactions. ${ }^{\mathbf{1 3 , 2 1 , 5 3 - 5 6}}$ In general, three types of metal-chromophore interactions will modulate the fluorescence intensities when a chromophore is in close proximity of a metal nanoparticle. First, local electric field amplification on the surface of metal nanoparticles will result in enhanced excitation efficiency if the excitation wavelength is resonant with the plasmon resonance. Second, the radiative decay rate of the chromophore will increase under the influence of plasmon resonance of metal nanoparticles. Third, non-radiative energy transfer will occur from the chromophore to the metal nanoparticle, which will introduce an additional non-radiative deactivation channel and result in a decreased fluorescence quantum yield of the chromophore. The fluorescence quantum yield is determined by the competition of the change in radiative and non-radiative decay rates. All three factors depend on the separation distance between the metal and chromophore. As the separation distance decreases, local electric field amplification increases monotonically, which contributes to an improved excitation efficiency. However, the competition of increased radiative and increased non-radiate decay rates will cause a complicated change in the fluorescence quantum yield. When a chromophore with a moderate quantum yield approaches a metal NP, the increase in the radiative decay rate plays a dominant role and its fluorescence quantum yield and intensity will generally increases first as the separation distance decreases until reaching an optimum separation distance. When the metalchromophore separation distance further decreases, the increase in the nonradiative energy transfer rate will play a dominant role and result in a decreased fluorescence quantum yield that may not be compensated by increased excitation efficiency (if any), then overall fluorescence intensity will decreases when the separation distance is too short. ${ }^{\mathbf{1 9 , 5 0 , 5 7}}$

The above trend was generally observed for a simple metalspacer-chromophore core-shell structure. The current system of $\mathrm{Ag} @ \mathrm{SiO}_{2}-\mathrm{Au}-\mathrm{RiTC}$ NPs is a little bit more complicated. Here RiTC is pre-quenched by the Au NPs in Au-RiTC NPs. Au-RiTC NP could be simply considered as a nano-chromophore entity with a much lower quantum yield compared to free RiTC molecules themselves. Their excitation efficiency and quantum yield will be modulated when they are attached to the surface of $\mathrm{Ag} @ \mathrm{SiO}_{2}$ NPs. Generally, a chromophore with a lower fluorescence quantum yield will experience larger enhancement in its fluorescence quantum yield. ${ }^{20}$ The fluorescence quantum yield of Au-RiTC NPs is then expected to experience a larger enhancement by Ag@SiO ${ }_{2}$ NPs. Furthermore, plasmon coupling will occur between the SPR bands of Au NPs and Ag NPs when $\mathrm{Au}-\mathrm{RiTC}$ NPs were attached to the surface of $\mathrm{Ag@SiO}$. Plasmon coupling between metal nanoparticles have been known to create giant electric field amplification in the gap region, which has been previously known to result in giant enhancement in SERS. ${ }^{30}$ RiTC molecules at the gap region of the $\mathrm{Ag} @ \mathrm{SiO}_{2}-\mathrm{Au}-$ RiTC NPs will experience a larger local electric field amplification (Fig. S10 $\dagger$ ), which provides extra enhancement in its excitation efficiency. The extra contribution due to plasmon coupling will cause extra fluorescence enhancement at shorter separation distances. To clarify plasmon coupling effects on the observed fluorescence enhancement, Ag@SiO ${ }_{2}-\mathrm{RiTC}$ NPs with different $\mathrm{SiO}_{2}$ shell thickness were prepared as the control experiments (Fig. S11 $\dagger$ ). The overall fluorescence of $\mathrm{Ag} @ \mathrm{SiO}_{2}-$ RiTC NPs was found quenched compared to free RiTC molecules. It has been previously reported that fluorescence of rhodamine molecules can be enhanced by Ag NP overlayers on the substrate. ${ }^{\mathbf{2 4 2 5}}$ Here the fluorescence quenching was observed in the colloid solution probably due to unfavorable spectral overlap between the SPR band of non-aggregated Ag NPs and RiTC as well as different sizes and aggregation states of Ag NPs, which are crucial to achieve fluorescence enhancement. ${ }^{24}$ Despite these differences, the relative fluorescence intensities of $\mathrm{Ag} @ \mathrm{SiO}_{2}-\mathrm{RiTC} \mathrm{NPs}$ and $\mathrm{Ag} @ \mathrm{SiO}_{2}-\mathrm{Au}-\mathrm{RiTC} \mathrm{NPs}$ displayed a similar trend: the fluorescence intensities increased first and then decreased as the $\mathrm{SiO}_{2}$ shell thickness decreased. The optimum fluorescence of $\mathrm{Ag} @ \mathrm{SiO}_{2}-\mathrm{RiTC}$ NPs was observed at a silica shell thickness of $20 \mathrm{~nm}$, which is larger than that for $\mathrm{Ag} @ \mathrm{SiO}_{2}-\mathrm{Au}-\mathrm{RiTC} \mathrm{NPs}(13 \mathrm{~nm})$. A thinner $\mathrm{SiO}_{2}$ thickness, i.e. smaller separation distance between Au NP and Ag NP, will result in stronger plasmon coupling between Au NP and Ag NP, and consequently larger local electric field amplification and further enhancement in the excitation efficiency. The optimum fluorescence of $\mathrm{Ag} @ \mathrm{SiO}_{2}-\mathrm{Au}-\mathrm{RiTC} \mathrm{NPs}$ is expected to occur at shorter separation distance compared to that of $\mathrm{Ag@SiO}{ }_{2}-\mathrm{RiTC}$, which is consistent with our experimental results. Furthermore, plasmon coupling in the $\mathrm{Ag} @ \mathrm{SiO}_{2}-\mathrm{Au}-\mathrm{RiTC}$ NPs structures is expected to exhibit stronger local electric field, ${ }^{38,52}$ and a larger excitation enhancement than that of $\mathrm{Ag} @ \mathrm{SiO}_{2}-\mathrm{RiTC}$ NPs. Consequently, larger fluorescence enhancements were observed for Ag@SiO ${ }_{2}-\mathrm{Au}-\mathrm{RiTC}$ NPs than that for Ag@SiO ${ }_{2}-\mathrm{RiTC}$ NPs for the same shell thickness. The optimum florescence intensity of $\mathrm{Ag} @ \mathrm{SiO}_{2}-\mathrm{Au}-\mathrm{RiTC}$ NPs was 101 times that of Au-RiTC NPs, 4.4 times that of free RiTC.

\section{Conclusions}

In summary, we have designed a coupled plasmonic nanostructure, Ag@SiO ${ }_{2}-\mathrm{Au}-\mathrm{RiTC} \mathrm{NPs}$, in which fluorescence of RiTC molecules that were pre-quenched by Au NPs were recovered or even enhanced through interactions with $\mathrm{Ag} @ \mathrm{SiO}_{2} \mathrm{NPs}$ $\mathrm{Ag} @ \mathrm{SiO}_{2}-\mathrm{Au}-\mathrm{RiTC} \mathrm{NPs}$ with a series of $\mathrm{SiO}_{2}$ shell thicknesses $(4,10,13,20,25,36,43$ and $50 \mathrm{~nm})$ have been prepared to optimize the fluorescence enhancement and investigate the enhancement mechanisms. $\mathrm{As}_{\mathrm{SiO}_{2}}$ shell thickness decreased, the fluorescence enhancement factor was found to increase first and then decrease for nanoparticles with very thinner $\mathrm{SiO}_{2}$ shell. The optimum fluorescence enhancement was found at $\mathrm{SiO}_{2}$ shell thickness of $13 \mathrm{~nm}$, at which the fluorescence intensity of $\mathrm{Ag} @ \mathrm{SiO}_{2}-\mathrm{Au}-\mathrm{RiTC}$ NPs was 101 times that of $\mathrm{Au}-$ RiTC NPs and 4.4 times that of free RiTC. The enhancement 
factors are much larger than that of the simple $\mathrm{Ag@SiO}{ }_{2}-\mathrm{RiTC}$ core-shell nanoparticle system with the same $\mathrm{SiO}_{2}$ shell thickness. The difference can be ascribed to extra enhancement in its excitation efficiency due to the plasmon coupling between $\mathrm{Au}$ $\mathrm{NP}$ and $\mathrm{Ag} \mathrm{NP}$ within each $\mathrm{Ag@SiO}{ }_{2}-\mathrm{Au}-\mathrm{RiTC} \mathrm{NP}$. Detection sensitivity of conventional fluorescence turn-on probes mostly relies on the contrast ratio between initial and quenched fluorescence intensity. The current strategy demonstrated a transition from strong fluorescence quenching to fluorescence enhancement, which could be utilized for development of fluorescence turn-on based detection platforms with enhanced contrast ratio and sensitivity.

\section{Acknowledgements}

This work is supported by the Ministry of Education, Singapore (Tier 1, R-143-000-607-112), the National Research Foundation, Prime Minister's Office, Singapore under its Competitive Research Program (CRP Award No. NRF-CRP10-2012-04), and the Guangdong Innovative Research Team Program of China (201101C0105067115).

\section{Notes and references}

1 L. Chen, D. W. McBranch, H.-L. Wang, R. Helgeson, F. Wudl and D. G. Whitten, Proc. Natl. Acad. Sci. U. S. A., 1999, 96, 12287-12292.

2 M. Fernandez-Suarez and A. Y. Ting, Nat. Rev. Mol. Cell Biol., 2008, 9, 929-943.

3 R. Jackson, R. P. Oda, R. K. Bhandari, S. B. Mahon, M. Brenner, G. A. Rockwood and B. A. Logue, Anal. Chem., 2014, 86, 1845-1852.

4 P. K. Chattopadhyay, D. A. Price, T. F. Harper, M. R. Betts, J. Yu, E. Gostick, S. P. Perfetto, P. Goepfert, R. A. Koup, S. C. De Rosa, M. P. Bruchez and M. Roederer, Nat. Med., 2006, 12, 972-977.

5 P. K. Chattopadhyay, T. M. Gierahn, M. Roederer and J. C. Love, Nat. Immunol., 2014, 15, 128-135.

6 D. Agard, Y. Hiraoka, P. Shaw and J. W. Sedat, Methods Cell Biol., 1989, 30, 353-377.

7 M. G. L. Gustafsson and W. W. Webb, Proc. Natl. Acad. Sci. U. S. A., 2005, 102, 13081-13086.

8 M. Gu, C. J. R. Sheppard and X. Gan, J. Opt. Soc. Am. A, 1991, 8, 1755-1761.

9 C. Fan, S. Wang, J. W. Hong, G. C. Bazan, K. W. Plaxco and A. J. Heeger, Proc. Natl. Acad. Sci. U. S. A., 2003, 100, 62976301.

10 M. Swierczewska, S. Lee and X. Chen, Phys. Chem. Chem. Phys., 2011, 13, 9929-9941.

11 D. Liu, S. Wang, M. Swierczewska, X. Huang, A. A. Bhirde, J. Sun, Z. Wang, M. Yang, X. Jiang and X. Chen, ACS Nano, 2012, 6, 10999-11008.

12 D. Liu, X. Huang, Z. Wang, A. Jin, X. Sun, L. Zhu, F. Wang, Y. Ma, G. Niu, A. R. Hight Walker and X. Chen, ACS Nano, 2013, 7, 5568-5576.

13 V. Giannini, A. I. Fernández-Domínguez, S. C. Heck and S. A. Maier, Chem. Rev., 2011, 111, 3888-3912.
14 H. Ehrenreich and H. R. Philipp, Phys. Rev., 1962, 128, 16221629.

15 K. L. Kelly, E. Coronado, L. L. Zhao and G. C. Schatz, J. Phys. Chem. B, 2003, 107, 668-677.

16 S. Link and M. A. El-Sayed, J. Phys. Chem. B, 1999, 103, 84108426.

17 P. Reineck, D. Gómez, S. H. Ng, M. Karg, T. Bell, P. Mulvaney and U. Bach, ACS Nano, 2013, 7, 6636-6648.

18 E. Dulkeith, A. C. Morteani, T. Niedereichholz, T. A. Klar, J. Feldmann, S. A. Levi, F. C. J. M. van Veggel, D. N. Reinhoudt, M. Möller and D. I. Gittins, Phys. Rev. Lett., 2002, 89, 203002.

19 D. Cheng and Q.-H. Xu, Chem. Commun., 2007, 248-250.

20 A. Kinkhabwala, Z. Yu, S. Fan, Y. Avlasevich, K. Muellen and

W. E. Moerner, Nat. Photonics, 2009, 3, 654-657.

21 C. Geddes and J. Lakowicz, J. Fluoresc., 2002, 12, 121-129.

22 K. Ray, R. Badugu and J. R. Lakowicz, J. Am. Chem. Soc., 2006, 128, 8998-8999.

23 B. B. Blizanac, P. N. Ross and N. M. Marković, J. Phys. Chem. $B, 2006,110,4735-4741$.

24 A. Suslov, P. T. Lama and R. Dorsinville, Opt. Commun., 2015, 345, 116-119.

25 S. Pan, Z. Wang and L. J. Rothberg, J. Phys. Chem. B, 2006, 110, 17383-17387.

26 K. Aslan, J. R. Lakowicz, H. Szmacinski and C. D. Geddes, J. Fluoresc., 2004, 14, 677-679.

27 O. G. Tovmachenko, C. Graf, D. J. van den Heuvel, A. van Blaaderen and H. C. Gerritsen, Adv. Mater., 2006, 18, 91-95.

28 L. Polavarapu, J. Perez-Juste, Q.-H. Xu and L. M. Liz-Marzan, J. Mater. Chem. C, 2014, 2, 7460-7476.

29 S. Nie and S. R. Emory, Science, 1997, 275, 1102-1106.

30 J. H. Lee, M. H. You, G. H. Kim and J. M. Nam, Nano Lett., 2014, 14, 6217-6225.

31 M. Rycenga, C. M. Cobley, J. Zeng, W. Li, C. H. Moran, Q. Zhang, D. Qin and Y. Xia, Chem. Rev., 2011, 111, 36693712 .

32 L. Zhou, F. Ding, H. Chen, W. Ding, W. Zhang and S. Y. Chou, Anal. Chem., 2012, 84, 4489-4495.

33 I. O. Osorio-Roman, A. R. Guerrero, P. Albella and R. F. Aroca, Anal. Chem., 2014, 86, 10246-10251.

34 M. D. Furtaw, D. L. Steffens, T. M. Urlacher and J. P. Anderson, Anal. Chem., 2013, 85, 7102-7108.

35 M. P. Busson, B. Rolly, B. Stout, N. Bonod and S. Bidault, Nat. Commun., 2012, 3, 962.

36 J. Zhang, Y. Fu, M. H. Chowdhury and J. R. Lakowicz, Nano Lett., 2007, 7, 2101-2107.

37 R. Gill, L. Tian, W. R. C. Somerville, E. C. Le Ru, H. van Amerongen and V. Subramaniam, J. Phys. Chem. C, 2012, 116, 16687-16693.

38 T. Zhang, N. Gao, S. Li, M. J. Lang and Q.-H. Xu, J. Phys. Chem. Lett., 2015, 6, 2043-2049.

39 M. Furtaw, J. Anderson, L. Middendorf and G. Bashford, Plasmonics, 2014, 9, 27-34.

40 L. Zhou, F. Ding, H. Chen, W. Ding, W. Zhang and S. Y. Chou, Anal. Chem., 2012, 84, 4489-4495.

41 G. Frens, Nature (London), Phys. Sci., 1973, 241, 20-22. 
42 D. S. Seferos, D. A. Giljohann, H. D. Hill, A. E. Prigodich and C. A. Mirkin, J. Am. Chem. Soc., 2007, 129, 15477-15479.

43 B. Wiley, Y. Sun, B. Mayers and Y. Xia, Chem.-Eur. J., 2005, 11, 454-463.

44 J. F. Hund, M. F. Bertino, G. H. Zhang, C. Sotiriou-Leventis, N. Leventis, A. T. Tokuhiro and J. Farmer, J. Phys. Chem. B, 2003, 107, 465-469.

45 E. M. Claesson and A. P. Philipse, Langmuir, 2005, 21, 94129419.

46 X. Qian, S. R. Emory and S. Nie, J. Am. Chem. Soc., 2012, 134, 2000-2003.

47 R. F. Chen, Arch. Biochem. Biophys., 1969, 133, 263-276.

48 Z. Guan, L. Polavarapu and Q.-H. Xu, Langmuir, 2010, 26, 18020-18023.
49 T. Ming, H. Chen, R. Jiang, Q. Li and J. Wang, J. Phys. Chem. Lett., 2012, 3, 191-202.

50 T. T. Zhao, K. Yu, L. Li, T. S. Zhang, Z. P. Guan, N. Y. Gao, P. Y. Yuan, S. Li, S. Q. Yao, Q.-H. Xu and G. Q. Xu, ACS Appl. Mater. Interfaces, 2014, 6, 2700-2708.

51 X.-F. Jiang, Y. Pan, C. Jiang, T. Zhao, P. Yuan, T. Venkatesan and Q.-H. Xu, J. Phys. Chem. Lett., 2013, 4, 1634-1638.

52 Z. Guan, N. Gao, X. F. Jiang, P. Yuan, F. Han and Q.-H. Xu, J. Am. Chem. Soc., 2013, 135, 7272-7277.

53 J. R. Lakowicz, Anal. Biochem., 2005, 337, 171-194.

54 W. L. Barnes, J. Mod. Opt., 1998, 45, 661-699.

55 T. V. Shahbazyan, Nano Lett., 2013, 13, 194-198.

56 R. F. Aroca, Phys. Chem. Chem. Phys., 2013, 15, 5355-5363.

57 P. Yuan, Y. H. Lee, M. K. Gnanasammandhan, Z. Guan, Y. Zhang and Q.-H. Xu, Nanoscale, 2012, 4, 5132-5137. 